\title{
No Association between p53 Immunohistochemical Staining and RASSF1 or DAPK1 Hypermethylation in Non-Small Cell Lung Cancer
}

\author{
Danny V. Colombara1 ${ }^{*}$, David Eidsmoe², Joshua E. Stern ${ }^{3}$, Qinghua Feng3 ${ }^{3}$ Hubert Vesselle², \\ Stephen E. Hawes ${ }^{4}$ \\ ${ }^{1}$ Institute for Health Metrics and Evaluation, University of Washington, Seattle, USA \\ ${ }^{2}$ Department of Radiology, University of Washington, Seattle, USA \\ ${ }^{3}$ Department of Pathology, University of Washington, Seattle, USA \\ ${ }^{4}$ Department of Epidemiology, University of Washington, Seattle, USA \\ Email: dvc2@uw.edu
}

Received 16 April 2015; accepted 25 July 2015; published 28 July 2015

Copyright (C) 2015 by authors and Scientific Research Publishing Inc.

This work is licensed under the Creative Commons Attribution International License (CC BY).

http://creativecommons.org/licenses/by/4.0/

(c) (i) Open Access

\begin{abstract}
p53 mutations have been linked with shortened survival rates in non-small cell lung cancer (NSCLC). Hypermethylation of RASSF1 and DAPK1 genes, which are downstream targets of p53, has also been linked to a poor prognosis in lung cancer patients. We investigated whether p53 mutations, assessed as p53 stabilization by immunohistochemistry (IHC), were independent of DAPK1 and RASSF1 promoter hypermethylation. We examined 103 resected NSCLC tumors for which we had p53 IHC and RASSF1 and DAPK1 methylation data. p53 protein expression was determined by IHC and graded using a semi-quantitative scoring method. DAPK1 and RASSF1 methylations were determined on tumor blocks by MethyLight real-time PCR assays represented as the percent of methylated reference DNA (PMR). Our primary results found no evidence for an association between the p53 IHC score and RASSF1 and DAPK1 PMR values, $P=0.46$ and $P=0.68$, respectively.
\end{abstract}

\section{Keywords}

NSCLC, p53, Hypermethylation, RASSF1, DAPK1

\footnotetext{
${ }^{*}$ Corresponding author.
}

How to cite this paper: Colombara, D.V., Eidsmoe, D., Stern, J.E., Feng, Q.H., Vesselle, H. and Hawes, S.E. (2015) No Association between p53 Immunohistochemical Staining and RASSF1 or DAPK1 Hypermethylation in Non-Small Cell Lung Cancer. Journal of Cancer Therapy, 6, 631-637. http://dx.doi.org/10.4236/jct.2015.68069 


\section{Introduction}

Lung cancer cases are predominantly comprised of four histological subtypes: small cell carcinoma, adenocarcinoma (ADC), squamous cell carcinoma (SCC), and large cell carcinoma (LCC). The latter three subtypes, which are collectively referred to as non-small cell lung cancers (NSCLC), have been the focus of a great deal of research.

p53, a tumor suppressor that inhibits cell proliferation by activating apoptotic pathways in abnormal or stressed cells, has been a common focus of NSCLC research [1]. p53 mediates transcription of regulatory genes through binding with promoter specific DNA sequences or interaction with other proteins for promoter methylation [2]. In damaged cells, p53 can repress downstream targets and allow repairs to be made before propagating errors that can lead to carcinogenesis [1]. Clinically, there is evidence that p53 mutations are associated with shortened survival times in NSCLC patients [3].

RASSF1 is a tumor suppressor gene that is inactivated through mutation or hypermethylation in the majority of human cancers, including NSCLC [4]. Clinically, there is evidence that RASSF1 has prognostic value for NSCLC [5]. Wildtype p53 binds to the RASSF1 promoter [1] [2] and recruits DNA methyltransferase 1 (DNMT1) and death-associated protein 6 (DAXX) [2], which leads to RASSF1 silencing through hypermethylation. It has been postulated that silencing of a tumor suppressor by another tumor suppressor might serve to balance between apoptosis and survival among damaged or stressed cells [1]. Due to the importance of RASSF1 among NSCLC cases, and its association with p53, we sought to assess whether p53 mutations were associated with RASSF1 promoter methylation.

DAPK1 is also a tumor suppressor that is often hypermethylated or otherwise inactivated in many human cancers, including NSCLC [6]. Clinically, DAPK1 promoter hypermethylation has been shown to have prognostic value among those with Stage 1 NSCLC [6]. DAPK1 is regulated by p53 through a binding site on its promoter [7]. Activation of p53 leads to increased expression of DAPK1 [7], with DAPK1 activating p53 through a p19ARF dependent mechanism, thereby forming a positive feedback loop [7].

In this analysis, we tested whether p53 mutation was independent of DAPK1 and RASSF1 promoter methylation.

\section{Materials and Methods}

\subsection{Study Population}

This is a secondary analysis of data from a previously published study of 18F-fluorodeoxyglucose (18F-FDG) uptake in primary NSCLC [8]. Briefly, the University of Washington Medical Center or the Veterans Affairs Puget Sound Health Care System diagnosed 208 participants with resectable primary NSCLC between February 1998 and August 2004. Promoter methylation of RASSF1 and DAPK1 was previously assessed in paraffin embedded tumor specimens [9].

\subsection{Pathology}

Pathologists from the University of Washington Medical Center or the Veterans Affairs Puget Sound Health Care System assessed biopsy and resection specimens in order to confirm NSCLC histology and to establish the histological sub-type: ADC, SCC, LCC, bronchoalveolar adenocarcinoma (BAC), and other/mixed NSCLC.

\subsection{Immunohistochemistry}

We considered elevated p53 protein levels, measured by immunohistochemistry (IHC), to be a surrogate marker of p53 mutation. However, not all functional p53 mutations lead to changes in p53 protein levels [10] and p53 protein may be elevated for reasons unrelated to mutation [11]. Despite the potential for misclassification, p53 protein level assessed by IHC continues to be used as a surrogate marker due its simplicity, robustness, and affordability [10] [11].

A detailed description of the IHC staining and scoring process has been previously described [12]. Briefly, due to heterogeneous IHC staining within tissue sections, we used a graded semi-quantitative scoring method for p53 IHC assessment in 103 specimens. Staining intensity was noted as 0, 1+, 2+, or 3+ and the percentage of cells staining at each intensity level was recorded. We calculated an intensity percentage score that ranged from 
zero (100\% of cells at 0 intensity) to 300 (100\% of cells at $3+$ intensity) [12]. For this assay, we used DO-7 monoclonal p53 antibodies (DAKO Corp., Carpinteria, CA) at 1:500 dilution.

\subsection{Promoter Methylation Assay}

The promoter methylation assay has been described in detail previously [4]. Briefly, DNA was isolated from 20 $\mu \mathrm{m}$ sections of paraffin embedded tumor blocks, digested with proteinase $\mathrm{K}$, and underwent phenol/chloroform extraction and ethanol precipitation. After sodium bisulfite conversion with appropriate controls, MethyLight real-time polymerase chain reaction (PCR) based assays were performed [13]. MethyLight data were reported as percent of methylated reference DNA (PMR) [14].

\subsection{Statistical Analysis}

In our primary analysis, the associations between p53 IHC data and RASSF1 and DAPK1 PMR were assessed using ordinary least squares regression. The association between the continuous forms of these variables was also assessed visually using LOWESS (locally weighted scatterplot smoothing) curves. In exploratory sub-analyses, RASSF1 and DAPK1 PMR were dichotomized at a threshold previously reported to represent biologically meaningful methylation $(\leq 4 \%,>4 \%$ ) [15]. Mann-Whitney U-tests and box plots were used to assess differences in the distribution of p53 IHC data by dichotomized RASSF1 and DAPK1 PMR. In sensitivity analyses, we dichotomized p53 in two ways and compared it with dichotomized RASSF1 and DAPK1. In the first method, we created a kernel density plot of the p53 IHC data to search for a natural p53 cut-point. In the second method, we used staining of $60 \%$ of the cells, regardless of the intensity, as the cut-point [16]. We considered P-values less than 0.05 statistically significant and we performed all analyses using Stata/SE 13.1 (StataCorp, College Station, TX).

\subsection{Ethics}

Informed consent in the parent study was obtained from all individual participants included in the study and the study was approved by the University of Washington Human Subjects Division [8].

\section{Results}

One-third of participants were 70+ years old, approximately one-third were 60 - 70 years old, and nearly onethird were younger than 60 years old (Table 1). Approximately $40 \%$ of participants were women and the majority (89\%) was white, with more than $70 \%$ reporting more than 40 pack-years of smoking history. Most tumors (65\%) were between two and five centimeters and were assessed as stage I (59\%) or II (25\%). The most common histological sub-types were ADC (40\%), SCC (35\%), and LCC (13.6) and poor differentiation was common (57\%).

p53 IHC staining intensity percentage scores were bimodal, with nearly 36\% having a score less than 100 and more than 57\% having a score of 201 - 300. The distribution of RASSF1 and DAPK1 PMR values were left skewed, with $93 \%$ and $100 \%$ having a PMR less than $34 \%$, respectively.

In our primary linear regression analysis, neither RASSF1 nor DAPK1 PMR was associated with p53 intensity percentage scores $(\mathrm{P}=0.46$ and $\mathrm{P}=0.68$, respectively). Visual examination of LOWESS curves of this data confirmed the lack of association (Figure 1).

There was no evidence of an association between p53 staining intensity percentage scores and dichotomized PMR of RASSF1 and DAPK1 (Mann-Whitney U-test P $=0.93$ and $\mathrm{P}=0.06$, respectively). Box plots confirmed the lack of association of p53 with DAPK1 and showed somewhat higher, though not statistically significant, p53 staining intensity with hypermethylated RASSF1 (Figure 2).

In sensitivity analyses, dichotomizing p53 at the lowest point of the bimodal distribution (0 - 180, 181 - 300) and by the percentage of cells staining at any intensity $(0 \%-59 \%, \geq 60 \%)$ did not change the null results reported above $(\mathrm{P}>0.05)$ (data not shown).

\section{Discussion}

We found that DAPK1 and RASSF1 promoter methylation was not associated with p53 IHC status. 
Table 1. Profile of 103 NSCLC patients and tumors.

\begin{tabular}{|c|c|c|}
\hline Characteristic & $\mathbf{N}$ & $(\%)$ \\
\hline \multicolumn{3}{|l|}{ Age } \\
\hline$<50$ & 4 & (3.9) \\
\hline $50-60$ & 27 & $(26.2)$ \\
\hline $60-70$ & 38 & (36.9) \\
\hline $70+$ & 34 & $(33.0)$ \\
\hline \multicolumn{3}{|l|}{ Sex } \\
\hline Female & 40 & (38.8) \\
\hline \multicolumn{3}{|l|}{ Race } \\
\hline African-American & 5 & (4.9) \\
\hline Asian & 4 & (3.9) \\
\hline White & 92 & $(89.3)$ \\
\hline Hispanic & 1 & $(1.0)$ \\
\hline Native American & 1 & $(1.0)$ \\
\hline \multicolumn{3}{|l|}{ Pack-years of tobacco } \\
\hline 0 & 4 & (3.9) \\
\hline $1-40$ & 22 & $(21.4)$ \\
\hline $40+$ & 74 & $(71.8)$ \\
\hline Smoker—amt. unknown & 3 & (2.9) \\
\hline \multicolumn{3}{|l|}{ Tumor size (cm) } \\
\hline $1-2$ & 28 & $(27.2)$ \\
\hline $2-3$ & 33 & $(32.0)$ \\
\hline $3-5$ & 34 & $(33.0)$ \\
\hline $5+$ & 8 & (7.8) \\
\hline \multicolumn{3}{|l|}{ Histologic stage } \\
\hline I & 61 & $(59.2)$ \\
\hline II & 26 & $(25.2)$ \\
\hline III & 14 & $(13.6)$ \\
\hline IV & 2 & (1.9) \\
\hline \multicolumn{3}{|l|}{ Histology sub-type } \\
\hline $\mathrm{ADC}$ & 41 & $(39.8)$ \\
\hline SCC & 36 & $(35.0)$ \\
\hline LCC & 14 & $(13.6)$ \\
\hline BAC & 6 & (5.8) \\
\hline NSCLC, other/mixed & 6 & (5.8) \\
\hline \multicolumn{3}{|l|}{ Differentiation } \\
\hline Well & 7 & (6.8) \\
\hline Moderate & 37 & (35.9) \\
\hline Poor & 59 & (57.3) \\
\hline
\end{tabular}



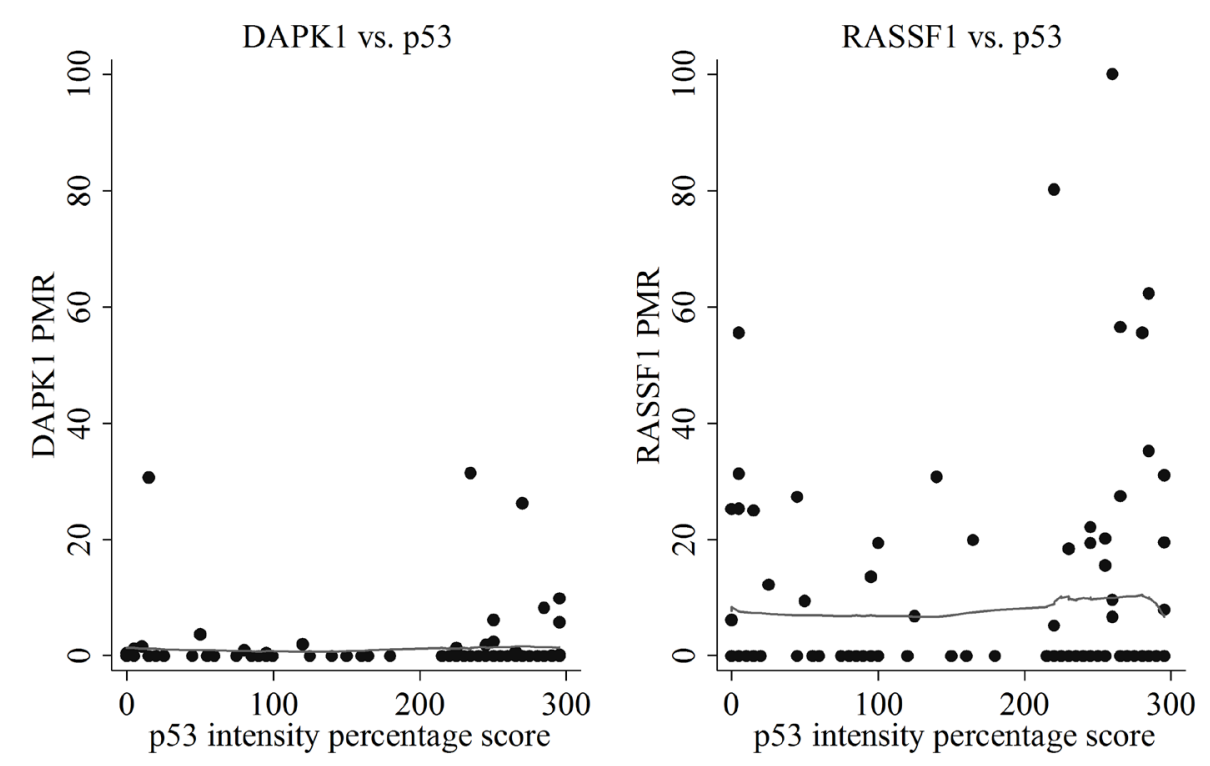

\section{LOWESS}

Figure 1. DAPK1 and RASSF1 percentage methylation reference (PMR) compared to p53 immunohistochemical staining intensity percentage scores.
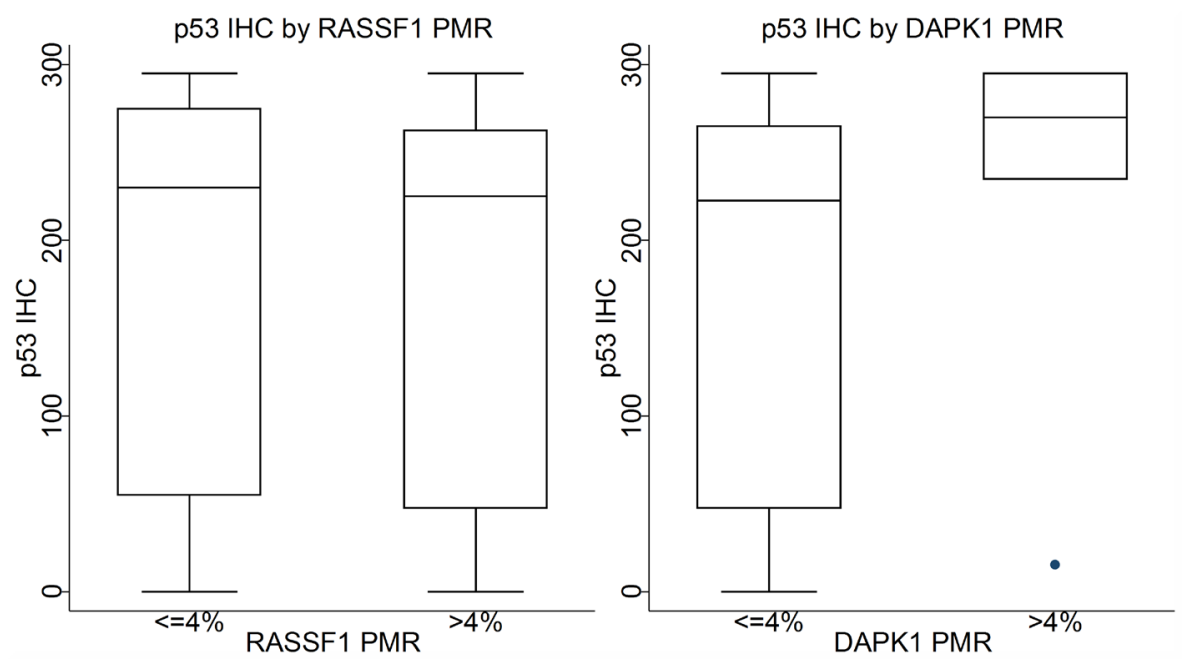

Figure 2. Box plots of p53 immunohistochemical staining intensity percentage scores by dichotomized RASSF1 and DAPK1 percentage methylation reference (PMR).

Martoriati et al. predicted that, due to a positive feedback loop, a mutation in DAPK1 would reduce the need for a subsequent p53 mutation leading to tumorigenesis [7]. Though we did not assess mutations in DAPK1, gene silencing through promoter methylation can be thought of as a functional equivalent. Our result is similar to that reported by Liu et al. [17], though p53 mutation status was assessed by sequencing by Liu's colleagues [17] and by IHC in our study.

The lack of association between p53 IHC and RASSF1 promoter methylation also agrees with Liu et al. [17]. However, it is contrary to the report of increased RASSF1A methylation associated with p53 mutation in a study of 206 Korean patients with NSCLC [18]. Based upon these conflicting results, it may be beneficial for additional studies to assess the relationship of p53 mutation with RASSF1 methylation.

The use of well-characterized cohort specimens is a strength of our study, as is the correspondence of our reported RASSF1 and DAPK1 methylation proportions with that in the literature [19] [20]. Nevertheless, our 
study also has limitations. As stated above, p53 IHC is an imperfect surrogate marker for functional p53 mutation [10] [11]. Therefore, we must proceed cautiously in comparing our results with those of prior studies that assessed p53 mutation using sequencing. Other limitations include insufficient statistical power to evaluate these associations by NSCLC histology sub-type and the lack of well-established cut-points for p53 IHC dichotomization.

\section{Conclusion}

In summary, in this study of 103 primary NSCLC tumors, we found no evidence of an association between p53 mutation (assessed by IHC staining) and DAPK1 and RASSF1 promoter hypermethylation.

\section{Acknowledgements}

We extend our gratitude to the participants in the 18F-fluorodeoxyglucose (18F-FDG) uptake in primary NSCLC study. This work was supported by the National Cancer Institute [CA115559 to HV, CA80907 to HV, CA107264 to HV, and R25 CA094880 trainee support to DVC]. The funders had no role in study design, data collection and analysis, decision to publish, or preparation of the manuscript.

\section{References}

[1] Tian, Y., Hou, Y., Zhou, X., et al. (2011) Tumor Suppressor RASSF1A Promoter: p53 Binding and Methylation. PLoS ONE, 6, e17017. http://dx.doi.org/10.1371/journal.pone.0017017

[2] Zhang, H., He, J., Li, J., et al. (2013) Methylation of RASSF1A Gene Promoter Is Regulated by p53 and DAXX. FASEB Journal, 27, 232-242. http://dx.doi.org/10.1096/fj.12-215491

[3] Murakami, I., Hiyama, K., Ishioka, S., et al. (2000) p53 Gene Mutations Are Associated with Shortened Survival in Patients with Advanced Non-Small Cell Lung Cancer: An Analysis of Medically Managed Patients. Clinical Cancer Research, 6, 526-530.

[4] Feng, Q., Hawes, S.E., Stern, J.E., et al. (2008) DNA Methylation in Tumor and Matched Normal Tissues from NonSmall Cell Lung Cancer Patients. Cancer Epidemiology, Biomarkers Prevention, 17, 645-654. http://dx.doi.org/10.1158/1055-9965.EPI-07-2518

[5] De Fraipont, F., Levallet, G., Creveuil, C., et al. (2012) An Apoptosis Methylation Prognostic Signature for Early Lung Cancer in the IFCT-0002 Trial. Clinical Cancer Research, 18, 2976-2986. http://dx.doi.org/10.1158/1078-0432.CCR-11-2797

[6] Tang, X., Khuri, F.R., Lee, J.J., et al. (2000) Hypermethylation of the Death-Associated Protein (DAP) Kinase Promoter and Aggressiveness in Stage I Non-Small-Cell Lung Cancer. Journal of the National Cancer Institute, 92, 15111516. http://dx.doi.org/10.1093/jnci/92.18.1511

[7] Martoriati, A., Doumont, G., Alcalay, M., et al. (2005) dapk1, Encoding an Activator of a p19ARF-p53-Mediated Apoptotic Checkpoint, Is a Transcription Target of p53. Oncogene, 24, 1461-1466.

http://dx.doi.org/10.1038/sj.onc.1208256

[8] Vesselle, H., Freeman, J.D., Wiens, L., et al. (2007) Fluorodeoxyglucose Uptake of Primary Non-Small Cell Lung Cancer at Positron Emission Tomography: New Contrary Data on Prognostic Role. Clinical Cancer Research, 13, 3255-3263. http://dx.doi.org/10.1158/1078-0432.CCR-06-1128

[9] Hawes, S.E., Stern, J.E., Feng, Q., et al. (2010) DNA Hypermethylation of Tumors from Non-Small Cell Lung Cancer (NSCLC) Patients Is Associated with Gender and Histologic Type. Lung Cancer, 69, 172-179. http://dx.doi.org/10.1016/j.lungcan.2009.11.002

[10] Kandioler-Eckersberger, D., Kappel, S., Mittlböck, M., et al. (1999) The TP53 Genotype but Not Immunohistochemical Result Is Predictive of Response to Cisplatin-Based Neoadjuvant Therapy in Stage III Non-Small Cell Lung Cancer. Journal of Thoracic and Cardiovascular Surgery, 117, 744-750. http://dx.doi.org/10.1016/S0022-5223(99)70295-3

[11] Robles, A.I. and Harris, C.C. (2010) Clinical Outcomes and Correlates of TP53 Mutations and Cancer. Cold Spring Harbor Perspectives in Biology, 2, Article ID: a001016. http://dx.doi.org/10.1101/cshperspect.a001016

[12] Brockenbrough, J.S., Morihara, J.K., Hawes, S.E., Stern, J.E., Rasey, J.S., Wiens, L.W., et al. (2009) Thymidine Kinase 1 and Thymidine Phosphorylase Expression in Non-Small-Cell Lung Carcinoma in Relation to Angiogenesis and Proliferation. Journal of Histochemistry and Cytochemistry, 57, 1087-1097. http://dx.doi.org/10.1369/jhc.2009.952804

[13] Weisenberger, D.J., Campan, M., Long, T.I., Kim, M., Woods, C., Fiala, E., et al. (2005) Analysis of Repetitive Element DNA Methylation by MethyLight. Nucleic Acids Research, 33, 6823-6836. http://dx.doi.org/10.1093/nar/gki987 
[14] Eads, C.A., Lord, R.V., Wickramasinghe, K., Long, T.I., Kurumboor, S.K., Bernstein, L., et al. (2001) Epigenetic Patterns in the Progression of Esophageal Adenocarcinoma. Cancer Research, 61, 3410-3418.

[15] Ogino, S., Kawasaki, T., Brahmandam, M., Cantor, M., Kirkner, G.J., Spiegelman, D., et al. (2006) Precision and Performance Characteristics of Bisulfite Conversion and Real-Time PCR (MethyLight) for Quantitative DNA Methylation Analysis. The Journal of Molecular Diagnostics, 8, 209-217. http://dx.doi.org/10.2353/jmoldx.2006.050135

[16] Yemelyanova, A., Vang, R., Kshirsagar, M., Lu, D., Marks, M.A., Shih, I.M. and Kurman, R.J. (2011) Immunohistochemical Staining Patterns of P53 Can Serve as a Surrogate Marker for TP53 Mutations in Ovarian Carcinoma: An Immunohistochemical and Nucleotide Sequencing Analysis. Modern Pathology, 24, 1248-1253. http://dx.doi.org/10.1038/modpathol.2011.85

[17] Liu, Y., Gao, W., Siegfried, J.M., Weissfeld, J.L., Luketich, J.D. and Keohavong, P. (2007) Promoter Methylation of RASSF1A and DAPK and Mutations of K-Ras, P53, and EGFR in Lung Tumors from Smokers and Never-Smokers. BMC Cancer, 7, 74. http://dx.doi.org/10.1186/1471-2407-7-74

[18] Lee, S.M., Lee, W.K., Kim, D.S. and Park, J.Y. (2012) Quantitative Promoter Hypermethylation Analysis of RASSF1A in Lung Cancer: Comparison with Methylation-Specific PCR Technique and Clinical Significance. Molecular Medicine Reports, 5, 239-244.

[19] Burbee, D.G., Forgacs, E., Zöchbauer-Müller, S., Shivakumar, L., Fong, K., Gao, B., et al. (2001) Epigenetic Inactivation of RASSF1A in Lung and Breast Cancers and Malignant Phenotype Suppression. Journal of the National Cancer Institute, 93, 691-699. http://dx.doi.org/10.1093/jnci/93.9.691

[20] Kim, D.H., Nelson, H.H., Wiencke, J.K., Christiani, D.C., Wain, J.C., Mark, E.J. and Kelsey, K.T. (2001) Promoter Methylation of DAP-Kinase: Association with Advanced Stage in Non-Small Cell Lung Cancer. Oncogene, 20, 17651770. http://dx.doi.org/10.1038/sj.onc.1204302 